\title{
Cigarette smoking and breast cancer
}

\author{
Kim Bennicke, Carsten Conrad, Svend Sabroe, Henrik Toft Sørensen
}

\begin{abstract}
Objective-To investigate cigarette smoking in relation to the risk of breast cancer.

Design-Interviews with women referred for mammography.

Setting-Hjørring District Hospital, a public, population based hospital in Denmark.

Subjects-3240 women aged 15-92 years referred for mammography during 1 June 1989 to 1 November 1991.

Main outcome measures-Smoking, parity, age, reason for referral, breast cancer.

Results-In a multiple logistic regression analysis adjusted for six variables a pronounced increased risk of breast cancer was found in women who had smoked for over 20 years, and this was significant in women who had smoked for over 30 years (odds ratio $1 \cdot 6,95 \%$ confidence interval $1 \cdot 1$ to $2 \cdot 3$ ). The median age of women with breast cancer was 59 (range 31-81) years for smokers and 67 (38-92) years for nonsmokers. The difference of eight years between the median ages was significant $(P<0 \cdot 01)$.

Conclusion-Smoking may increase the risk of breast cancer after 30 years of smoking.
\end{abstract}

\section{Introduction}

Breast cancer is the most common cancer in women throughout the world, and its incidence is increasing. The prognosis after treatment has not improved greatly during the past few decades. There is therefore a considerable demand to identify possible risk factors. Two hypotheses of the relation between smoking and breast cancer have been suggested. On the basis of the finding of reduced urinary oestrogen during the luteal phase of the menstrual cycle it has been suggested that cigarette smoking may reduce the risk of breast cancer. ${ }^{12}$ On the basis of the finding of mutagens from cigarette smoke in the breast fluid of non-lactating women a contrary hypothesis has been suggested-that tobacco smoke may have a direct carcinogenic effect. ${ }^{34}$ Several studies have examined the relation between cigarette smoking and breast cancer, usually on the basis of the hypothesis of a lower risk of cancer among smokers than non-smokers, but the results of the studies have been contradictory. ${ }^{5}$ We investigated the relation between smoking and the risk of breast cancer in women referred for mammography.

\section{Subjects and methods}

We studied all women $(n=3240)$ referred for mammography to the department of radiology, Hjørring District Hospital, Denmark, from 1 June 1989 to 1 November 1991. The hospital is a public, population based hospital with a well defined catchment area.

\section{SMOKING AND OTHER RISK FACTORS}

Before mammography we interviewed all the women who were referred for the test and recorded clinical information and data on smoking habits, number of births, breast feeding, and age. Before any diagnoses of cancer were made the women were divided into exposure groups according to their smoking habits. We recorded the number of cigarettes each woman smoked a day (1-5, 6-10,11-15, 16-20, and >20) and the number of years she had been a smoker $(1-5,6-10$, 11-20, 21-30, and >30). We did not distinguish between smokers and former smokers at the time of examination.

The two major symptoms that led to referral were a palpable tumour in the breast (in 1241 women) and localised tenderness (in 1057); 125 women were referred because of cancerphobia, although no symptoms were present.

\section{OUTCOME VARIABLE}

The mammography comprised two standard views, plus a craniocaudal view when necessary. Clinical examination was by inspection and palpation of the breast and armpit. Depending on the findings of the mammography and the clinical examination, a woman might also have had one or more of the following procedures: fine needle aspiration, ductography, or aerography. We obtained information about the final diagnosis of breast cancer from the hospital records and the department of pathology.

\section{STATISTICAL METHODS}

Bivariate relations were evaluated with $\chi^{2}$ tests for independence within contingency tables and with the Mann-Whitney U test; when multiple variables were considered, multiple logistic regression was used (spss/ PC, Advanced Statistics. Version 4.0). The cumulative risk was estimated with odds ratios (with $95 \%$ confidence intervals). Potential confounding variables were tested in multivariate analyses; if they changed the estimate of the association of interest, they remained in the final model. ${ }^{6}$

\section{Results}

Of the 3240 women who had mammography (median age 45 (range 15-92) years), 1828 were smokers (median age $44(15-86)$, and 1412 were non-smokers (median age 45 (19-92)). Table I shows the age distribution. In all, 230 cases of cancer were diagnosed (127 among the smokers, 103 among the non-smokers), of which 223 were carcinomas; in the other seven cases the women had radiation therapy without surgery. Of the women with cancer, 198 had given birth, of whom 176 had breast fed their children and $111(58 \%)$ were smokers (12 of the $32(38 \%)$ nulliparous women were smokers).

The prevalence of breast cancer in the non-smokers was $7 \%$. Prevalence in women who had been smoking for 1-30 years ranged from $3 \%$ to $6 \%$ (overall $4 \%$ $(55 / 1347))$, while in those who had been smoking for more than 30 years it was $15 \%(71 / 463)$. The relation 
between breast cancer and parity was ambiguous, with the highest prevalence of cancer being found in the groups with one and over four deliveries.

The prevalence of breast cancer varied with respect to the other competing risk factors. Among women who had breast fed and among those with a family history of breast cancer it was 7\% (176/2417) and $8 \%$ (52/679) respectively, while among women who had undergone gynaecological operations it was $11 \%$ (9/83). Parity and gynaecological operations are cumulated measurements, closely correlated with age.

Table II shows the result of a logistic regression analysis including six variables, of which only age and a history of $>30$ years' smoking gave a significantly increased risk of developing breast cancer. As breast feeding, family history of breast cancer, and previous gynaecological operations did not significantly alter the risk estimate for smoking, these variables were not included in the final model.

The logistic regression analysis showed a slightly increased risk of breast cancer in women with one delivery. The greatly increased risk for multiparous women that was seen in the bivariate analyses disappeared after adjustment for age. A pronounced increased risk of breast cancer was seen in women who had smoked for over 20 years. Using trend analysis we found an increasing odds ratio of $1 \cdot 14$ with each smoking category.

The median age of the women with cancer was 59 (range 31-86) years among those who smoked and 67 (38-92) among those who did not. The difference in median age was significant $(P<0.01)$.

\section{Discussion}

We have shown that, after adjustment for parity, age, family history of breast cancer, and breast feeding, women who have smoked for more than 30 years have a significantly increased risk of breast cancer, with a relative risk of $1 \cdot 6$. The study also showed that women with breast cancer who smoked were eight years younger than those who did not smoke. The study thus suggests that smoking is only a small risk factor for developing breast cancer, but that because of the earlier development of breast cancer in smokers, it probably has an effect modifying influence. The published studies of smoking and breast cancer are inconsistent in their conclusions, and our study does not support the hypothesis that smoking has a protective influence on developing breast cancer because of its anti-oestrogenic effect.

\section{SELECTION BIAS}

Selection bias is not likely for several reasons. Danish hospitals cover the whole population, and hospital treatment is free. All women referred for mammography from the area covered by a particular hospital have it performed in the radiology department of that hospital. All women in whom breast cancer is suspected and all those with a palpable tumour in the breast have preoperative mammography. In the Danish hospital register, which contains all the discharge diagnoses from all hospitals in Denmark, we found no patients with breast cancer from our area who were not included in our study.

Four different hypotheses might explain the finding that women with breast cancer who smoked were significantly younger than those who did not smoke. Firstly, if smoking is considered a risk factor by doctors and patients then smokers might be referred for mammography more often and at a younger age. However, the prevalence of smokers and former smokers in our population $(56 \%)$ corresponded with the prevalence in the population as a whole. ${ }^{7}$ Consequently, no indications suggest that smokers are
TABLE I-Age distribution of 3240 women referred to Hjorring District Hospital for mammography, by smoking and breast cancer status

\begin{tabular}{lccccc}
\hline & \multicolumn{2}{c}{ Smokers } & & \multicolumn{2}{c}{ Non-smokers } \\
\cline { 2 - 3 } \cline { 5 - 6 } $\begin{array}{l}\text { Age group } \\
\text { (years) }\end{array}$ & Total No & $\begin{array}{c}\text { No (\%) with } \\
\text { breast cancer }\end{array}$ & & Total No & $\begin{array}{c}\text { No (\%) with } \\
\text { breast cancer }\end{array}$ \\
\hline$<29$ & 216 & $0(0)$ & & 152 & $0(0)$ \\
$30-39$ & 480 & $11(2 \cdot 3)$ & & 294 & $2(0 \cdot 7)$ \\
$40-49$ & 548 & $26(4 \cdot 7)$ & & 424 & $22(5 \cdot 2)$ \\
$50-59$ & 355 & $29(8 \cdot 2)$ & & 272 & $15(5 \cdot 5)$ \\
$60-69$ & 154 & $31(20 \cdot 1)$ & & 137 & $20(14 \cdot 6)$ \\
$70-79$ & 61 & $21(34 \cdot 4)$ & & 88 & $21(23 \cdot 9)$ \\
$\geqslant 80$ & 14 & $9(64 \cdot 3)$ & & 45 & $23(51 \cdot 1)$ \\
\hline Total & 1828 & $127(6 \cdot 9)$ & & 1412 & $103(7 \cdot 3)$ \\
\hline
\end{tabular}

TABLE II-Logistic regression analysis with breast cancer as dependent variable

\begin{tabular}{|c|c|c|}
\hline & Full model & Reduced model \\
\hline Variable & $\begin{array}{l}\text { Odds ratio ( } 95 \% \\
\text { confidence interval) }\end{array}$ & $\begin{array}{l}\text { Odds ratio ( } 95 \% \\
\text { confidence interval) }\end{array}$ \\
\hline \multicolumn{3}{|l|}{ Parity: } \\
\hline 0 & 1.0 & 1.0 \\
\hline 1 & $1.9(1.0$ to 3.6$)$ & $1.8(1.1$ to 3.0$)$ \\
\hline 2 & $0.9(0.5$ to 1.8$)$ & \\
\hline 3 & $1 \cdot 1(0.5$ to $2 \cdot 1)$ & $1.0(0.6$ to 1.5$)$ \\
\hline$\geqslant 4$ & $1 \cdot 1(0.5$ to $2 \cdot 1)$ & \\
\hline \multicolumn{3}{|l|}{ Smoking (No of years): } \\
\hline 0 & $1 \cdot 0$ & $1 \cdot 0$ \\
\hline $1-10$ & $1.1(0.6$ to 2.4$)$ & $1.1(0.6$ to $2 \cdot 1)$ \\
\hline $11-20$ & $0.9(0.5$ to 1.7$)$ & $0.9(0.5$ to 1.7$)$ \\
\hline $21-30$ & $1 \cdot 3(0 \cdot 8$ to $2 \cdot 1)$ & $1.3(0.7$ to 1.9$)$ \\
\hline$\geqslant 31$ & $1.6(1 \cdot 1$ to $2 \cdot 2)$ & $1.6(1 \cdot 1$ to $2 \cdot 3)$ \\
\hline \multicolumn{3}{|l|}{ Age (years): } \\
\hline$<29$ & $1 \cdot 0$ & $1 \cdot 0$ \\
\hline$\geqslant 30$ (10yearagegroups) & $2 \cdot 3(2 \cdot 0$ to $2 \cdot 5)$ & $2 \cdot 3(2 \cdot 0$ to $2 \cdot 5)$ \\
\hline \multicolumn{3}{|l|}{ Breast feeding: } \\
\hline No & $1 \cdot 0$ & \\
\hline Yes & $1.0(0.6$ to 1.6$)$ & \\
\hline \multicolumn{3}{|c|}{ Family history of breast cancer: } \\
\hline No & $1 \cdot 0$ & \\
\hline Yes & $1.1(0.8$ to 1.6$)$ & \\
\hline \multicolumn{3}{|l|}{ Gynaecological operation: } \\
\hline No & $1 \cdot 0$ & \\
\hline Yes & $0.7(0.3$ to 1.5$)$ & \\
\hline
\end{tabular}

referred for mammography more often than nonsmokers.

Secondly, if smoking is considered a risk factor for breast cancer then smokers might be better examined by the doctors, and the probability of finding a small breast tumour would therefore be greater; finding a tumour leads to referral for mammography. In our study, however, the prevalence of palpable tumours was identical in smokers and non-smokers; a comparison of these facts with the age distribution of the two groups does not suggest that smokers would be referred for mammography at an earlier stage than non-smokers and therefore would be younger when cancer was diagnosed.

Thirdly, the population of smokers is much younger than that of non-smokers. The distribution of smokers in the various age groups, however, was the same in the study population as in the population as a whole.

Fourthly, cigarette smoking is a carcinogenic factor leading to breast cancer at an earlier age in smokers than in non-smokers.

\section{REPORTING OF SMOKING HABITS}

Information about smoking habits was obtained by interview. Several biochemical validity studies have shown that self reported smoking habits are good measurements for a population's habits, although the possibility of some underreporting cannot be excluded. ${ }^{89}$

\section{UNLIKELY CONFOUNDERS}

The comprehensive differences in the incidence of breast cancer, as well as the increasing incidence, suggest that environmental factors are responsible for a large proportion of breast cancer. Several factors other 


\section{Key messages}

- Breast cancer is the commonest cancer in women throughout the world, and its incidence is increasing

- It has been suggested that smoking protects against breast cancer because of an antioestrogenic effect

- This study shows, however, that women who have smoked for more than 30 years have an increased risk of developing breast cancer

- Moreover, smokers with breast cancer were eight years younger than non-smokers with breast cancer who had smoked for more than 30 years. Moreover, breast cancer will occur eight years earlier in smokers than in non-smokers. This finding could support the hypothesis of a direct carcinogenic effect of tobacco.

This study was financially supported by the Niels Jensen Foundation and Hjørring District Hospital.

1 MacMahon B, Trichopoules D, Cole P, Brown J. Cigarette smoking and urinary estrogens. N Engl F Med 1982;307:1062-5.

2 Baron JA, LaVecchia C, Levi F. The antiestrogenic effect of cigarette smoking in women. Am f Obstet Gynecol 1990;162:502-14.

3 Petrakis NL, Maack CA, Lee RE, Jyon M. Mutagenic activity in nipple aspirates of human breast fluid. Cancer Res 1980;40:188-9.

4 Hiatt RA, Fireman BH. Smoking, menopause, and breast cancer. $f$ Natl Cancer Inst 1986;76:833-8.

5 Palmer JR, Rosenberg L. Cigarette smoking and the risk of breast cancer. Epidemiol Rev 1993;5:145-56.

than those included in our model-for example, alcohol, diet, body size, and use of oral contraception -have been studied extensively. ${ }^{10-12}$ The associations between these factors and breast cancer, however, are often weak and inconsistent. Although confounding cannot be ruled out as an explanation of our results, it is not likely that an inverse relation exists between smoking and breast cancer.

\section{CONCLUSION}

In conclusion, nothing in the present study suggests that smoking protects against breast cancer-rather, the opposite. Smoking seemed to increase the risk of developing cancer to a modest extent among women
6 Hosmer DW, Lemeshow S. Applied logistic regression. New York: John Wiley, 1989.

7 Osler M. Danskernes rygevaner [Smoking habits of Danes]. Dansk Institut for Klinisk Epidemiologi: Copenhagen, 1992.

8 Krall EA, Valadian I, Dwyer JT, Gardener J. Accuracy of recalled smoking

9 Vogt TM, Steve S, Widdenson G, Hulley SB. Expired air carbon monoxide and serum thiocyanate as objective measures of cigarette consumption. $\mathrm{Am} \mathcal{F}$ Public Health 1977;67:545-9.

10 Hunter DJ, Willett WC. Diet, body size, and breast cancer. Epidemiol Rev 1993;15:110-32.

11 Malone KE, Daling JR, Weiss NS. Oral contraceptives in relation to breast cancer. Epidemiol Rev 1993;15:80-97.

12 Roth HD, Levy PS, Shi L, Post E. Alcoholic beverages and breast cancer: some observations on published case-control studies. $f$ Clin Epidemiol 1994;47:207-16.

(Accepted 11 April 1995) data. Am $\mathcal{F}$ Public Health 1989;79:200-6.

\title{
Treatment discontinuation with selective serotonin reuptake inhibitors compared with tricyclic antidepressants: a meta-analysis
}

\author{
I M Anderson, B M Tomenson
}

\begin{abstract}
Objective-To assess treatment discontinuation rates with selective serotonin reuptake inhibitors compared with tricyclic antidepressants.

Design-Meta-analysis of 62 randomised controlled trials.

Subjects -6029 patients with major unipolar depression.

Main outcome measures-Pooled risk ratios for drop out rates with respect to all cases of discontinuation and those due to side effects and treatment failure.

Results-The total discontinuation rate was $10 \%$ lower with selective serotonin reuptake inhibitors than with tricyclic antidepressants (risk ratio 0.90; $95 \%$ confidence interval 0.84 to 0.97 ) and the drop out rate due to side effects was $25 \%$ lower (risk ratio $0.75 ; 0.66$ to 0.84 ). There was no significant difference between drug classes in the drop out rates for treatment failure. The risk ratios for drop out did not differ significantly between individual selective serotonin reuptake inhibitors.

Conclusions-Selective serotonin reuptake inhibitors are better tolerated than tricyclic antidepressants as measured by total numbers of drop outs. The definite advantage to selective serotonin reuptake inhibitors is explained by fewer drop outs due to side effects. The overall difference, however, is comparatively small and may not be clinically relevant. Analyses of cost effectiveness should not overestimate the advantage to selective serotonin reuptake inhibitors.
\end{abstract}

I M Anderson, senior lecturer

B M Tomenson, statistician

Correspondence to:

Dr Anderson.

$B M \Im$ 1995;310:1433-8
There is debate about the cost effectiveness of newer, more expensive antidepressants-especially the selective serotonin reuptake inhibitors-compared with the cheaper, first generation tricyclic antidepressants. ${ }^{12}$ Both groups of drugs are of comparable efficacy, ${ }^{3-5}$ so that cost effectiveness hinges on tolerability and safety. Tricyclic antidepressants are much cheaper per tablet than selective serotonin reuptake inhibitors. But if compliance is poorer tricyclic antidepressants may prove more expensive overall because of costs incurred through treatment failure (for example, costs of extra treatment, time off work, sickness benefit, etc) ${ }^{6}$

One way of assessing relative tolerability is to measure discontinuation rates in randomised, double blind, parallel comparative trials. Though this is fairly crude, ${ }^{7}$ it provides an objective measure of the proportion of patients who persist with each treatment during the study. In an influential meta-analysis, Song et al found that efficacy and drop out rates did not differ between tricyclic antidepressants and selective serotonin reuptake inhibitors and concluded that the increasing use of the newer drugs as first line treatment was not warranted. ${ }^{4}$ Their analysis was weakened by the inclusion of non-tricyclic, second generation antidepressants in the tricyclic group (table I), accounting for about a quarter of the studies they analysed. These drugs generally have a better side effect profile than the tricyclic antidepressants ${ }^{8}$ but are infrequently used in practice. ${ }^{9}$ Hence the clinical relevance of their inclusion in the meta-analysis can be questioned.

Montgomery et al confined their meta-analysis to studies including tricyclic antidepressants and found that selective serotonin reuptake inhibitors caused significantly fewer drop outs due to side effects whereas there was no difference in drop outs due to inefficacy. ${ }^{7}$ Unfortunately, given that discontinuation due to side effects accounts for only $30-50 \%$ of drop outs, ${ }^{4727}$ they failed to analyse total drop out rates. 